\title{
MULTIPLICITY OF NODAL SOLUTIONS FOR ELLIPTIC EQUATIONS WITH SUPERCRITICAL EXPONENT IN CONTRACTIBLE DOMAINS
}

\author{
Donato Passaseo
}

\section{Introduction}

This paper is concerned with existence and multiplicity of solutions for the problem

$$
P(\Omega, p) \begin{cases}\Delta u+|u|^{p-2} u=0 & \text { in } \Omega, \\ u=0 & \text { on } \partial \Omega, \\ u^{+} \not \equiv 0, \quad u^{-} \not \equiv 0 & \text { in } \Omega,\end{cases}
$$

where $\Omega$ is a bounded domain in $\mathbb{R}^{n}$, with $n \geq 3$ and $p>2^{*}=2 n /(n-2)\left(2^{*}\right.$ is the critical exponent for the Sobolev embedding $H_{0}^{1,2}(\Omega) \hookrightarrow L^{p}(\Omega)$ ). Following the notation introduced in [3], we shall refer to the solutions of $P(\Omega, p)$ as nodal solutions.

It is well known that the main difficulty in problems of this type is related to the lack of compactness due to the presence of the exponent $p>2 n /(n-2)$. Therefore the classical topological methods of calculus of variations cannot be applied in a straightforward way. When $2<p<2 n /(n-2)$, the existence of positive and nodal solutions for the problem

$$
\begin{cases}\Delta u+|u|^{p-2} u=0 & \text { in } \Omega, \\ u=0 & \text { on } \partial \Omega, \\ u \neq \equiv 0 & \text { in } \Omega,\end{cases}
$$

1991 Mathematics Subject Classification. 35J65, 35J20. 
does not depend on the shape of $\Omega$; on the contrary, when $p \geq 2 n /(n-1)$, no solution can exist if $\Omega$ is starshaped, as a consequence of the well known Pokhozhaev identity (see [36]). The solvability of problem $(*)$ when $p \geq 2 n /(n-2)$ seems to be strictly related to the shape of $\Omega$ : if $p=2 n /(n-2)$, important results of Bahri and Coron (see [4], [15]) guarantee the existence of positive solutions for (*) in domains $\Omega$ having nontrivial topology (in a suitable sense); afterwards existence and multiplicity results of positive solutions have been stated even in some contractible domains (see [16], [17], [27], [28], [31]).

Problem $(*)$ with $p>2 n /(n-2)$ has been studied in [33]-[35]: in [33] and [34] one can find examples of domains $\Omega$, nontrivial in the sense of Bahri-Coron [4], where problem $(*)$ has no solution for $p>2 n /(n-2)$; on the other hand, in [35] it is proved that for all $p>2 n /(n-2)$ one can find contractible domains where the number of positive solutions of $(*)$ is arbitrarily large (other problems with supercritical nonlinearity are studied in [22]-[25]).

In [8] Brezis and Nirenberg showed that some perturbations of the equation by lower order terms can guarantee the existence of positive solutions, independently of the shape of the domain. Afterwards many authors (see [2], [10]-[12], [18], [43], [39]) studied existence and multiplicity of nontrivial solutions for the problem

$$
P_{\lambda}(\Omega) \begin{cases}\Delta u+\lambda u+|u|^{p-2} u=0 & \text { in } \Omega, \\ u=0 & \text { on } \partial \Omega, \\ u \neq 0 & \text { in } \Omega,\end{cases}
$$

with $p=2 n /(n-2)$ and $\lambda>0$. In particular, nodal solutions are obtained in [12].

A nonexistence result for the nodal solutions of $P_{\lambda}(\Omega)$ is stated in [3]: if $\Omega$ is a ball of $\mathbb{R}^{n}$ with $n=4,5,6$, then there exists $\lambda^{*}>0$ such that $P_{\lambda}(\Omega)$ has no radial solution for $\lambda<\lambda^{*}$; on the contrary, for $n \geq 7$, in [39] and [12] it is proved that there exist infinitely many radial nodal solutions of $P_{\lambda}(\Omega)$ for all $\lambda>0$.

Problem $P_{\lambda}(\Omega)$, with $\lambda=0$ and $p=2 n /(n-2)$, is studied in [21], where the existence of a nodal solution of $P_{0}(\Omega)$ is proved in some symmetric domains (the same as those considered in [27]).

In the present paper we show that for every $p>2 n /(n-2)$ there exist contractible domains $\Omega$ (the same as those introduced in [27] and [35]) such that problem $P(\Omega, p)$ has nodal solutions: for all $h \in \mathbb{N}$ we find a bounded domain $D_{h}$ and $h$ perturbations, obtained by removing $h$ subsets $\chi_{1}, \ldots, \chi_{h}$ of small capacity, such that $D_{h} \backslash \bigcup_{i=1}^{h} \chi_{i}$ is a contractible domain and the problem $P\left(D_{h} \backslash \bigcup_{i=1}^{h} \chi_{i}, p\right)$ has at least $h^{2}$ nodal solutions.

Indeed, for any fixed sets $\chi_{r}$ and $\chi_{s}$ (not necessarily distinct), we find a solution $u$ of $P\left(D_{h} \backslash \bigcup_{i=1}^{h} \chi_{i}, p\right)$ such that $u^{+}$and $u^{-}$concentrate near $\chi_{r}$ and 
$\chi_{s}$ respectively as their capacity tends to zero; therefore different pairs $\left(\chi_{r}, \chi_{s}\right)$ give rise to different solutions and so we have on the whole $h^{2}$ nodal solutions.

As in [12] and in [21], an important role in finding the nodal solutions is played by the set

$$
M=\left\{u \in H_{0}^{1,2}(\Omega): u^{ \pm} \not \equiv 0, f^{\prime}(u)\left[u^{ \pm}\right]=0\right\}
$$

Notice that $M$ is not a smooth submanifold of $H_{0}^{1,2}(\Omega)$; therefore we shall use a special device, based on a result of C. Miranda (see [26]).

Since the arguments used in [27] and in [21] when $p=2 n /(n-2)$ do not apply when $p>2 n /(n-2)$ (see Remark 3.4), in this paper we introduce suitable obstacles, which, combined with the symmetry properties of the domains, allow us to overcome the difficulties given by the lack of compactness, due to the presence of the supercritical nonlinearities.

The main result obtained in this paper is stated in Theorem 2.2; some qualitative properties of the solutions obtained are described in Proposition 3.2.

In the next section we recall some notions of "subdifferential calculus", which we shall use in studying problems with obstacle.

\section{Preliminary results}

In this section we specify the variational framework of $P(\Omega, p)$ : solving this problem is equivalent to finding critical points of a functional on a suitable constraint. Moreover, we recall the notion and some properties of the subdifferential (see, for instance, [13], [14]), which we shall use in next sections.

The solutions of $P(\Omega, p)$ are the critical points of the functional $f: H_{0}^{1,2}(\Omega) \cap$ $L^{p}(\Omega) \rightarrow \mathbb{R}$ defined by

$$
f(u)=\frac{1}{2} \int_{\Omega}|D u|^{2} d x-\frac{1}{p} \int_{\Omega}|u|^{p} d x,
$$

which belong to

$$
\begin{aligned}
M=\left\{u \in H_{0}^{1,2}(\Omega) \cap L^{p}(\Omega): u^{+} \not \equiv 0,\right. & u^{-} \not \equiv 0, \\
& \left.f^{\prime}(u)\left[u^{+}\right]=f^{\prime}(u)\left[u^{-}\right]=0\right\} .
\end{aligned}
$$

The space $H_{0}^{1,2}(\Omega) \cap L^{p}(\Omega)$ is endowed with the norm

$$
\|u\|=\left(\int_{\Omega}|D u|^{2} d x\right)^{1 / 2}+\left(\int_{\Omega}|u|^{p} d x\right)^{1 / p}
$$

in such a way that $f$ is a $C^{2}$-functional in $H_{0}^{1,2}(\Omega) \cap L^{p}(\Omega)$. 
Definition 1.1. Let $X$ be a normed vector space and $\Phi: X \rightarrow \mathbb{R} \cup\{\infty\}$ be a given function. We shall call the set $D(\Phi)=\{u \in X: \Phi(u)<\infty\}$ the domain of $\Phi$. For all $u \in D(\Phi)$ we define the subdifferential of $\Phi$ at $u$ to be the set $\partial^{-} \Phi(u)$ consisting of all $\alpha$ in $X^{\prime}$ (the dual space of $X$ ) such that

$$
\liminf _{v \rightarrow u} \frac{\Phi(v)-\Phi(u)-\alpha(v-u)}{\|v-u\|} \geq 0 .
$$

$\Phi$ is said to be subdifferentiable at $u$ if $\partial^{-} \Phi(u) \neq \emptyset$; moreover, we say that $u$ is a lower critical point for $\Phi$ if $0 \in \partial^{-} \Phi(u)$.

EXAMPLES 1.2. It is easy to verify that, if $X=H_{0}^{1,2}(\Omega) \cap L^{p}(\Omega)$ is endowed with the norm (1.3) and if $g(u)=\int_{\Omega}|D u|^{2} d x$, then $\partial^{-} f(u)=\left\{f^{\prime}(u)\right\}$ and $\partial^{-} g(u)=\left\{g^{\prime}(u)\right\}$ where

$$
\begin{aligned}
& f^{\prime}(u)[v]=\int_{\Omega} D u D v d x-\int_{\Omega}|u|^{p-2} u v d x, \\
& g^{\prime}(u)[v]=\int_{\Omega} D u D v d x \quad \forall v \in H_{0}^{1,2}(\Omega) \cap L^{p}(\Omega) .
\end{aligned}
$$

For every subset $E$ of $X$, let $I_{E}$ be the function defined by

$$
I_{E}(u)= \begin{cases}0 & \text { if } u \in E, \\ \infty & \text { if } u \in X \backslash E .\end{cases}
$$

If $u \in V=\left\{u \in H_{0}^{1,2}(\Omega): \int_{\Omega}|u|^{p} d x=1\right\}$, then one can verify that $\alpha \in \partial^{-} I_{V}(u)$ if and only if there exists $\lambda \in \mathbb{R}$ such that

$$
\alpha(v)=\lambda \int_{\Omega}|u|^{p-2} u v d x \quad \forall v \in H_{0}^{1,2}(\Omega) \cap L^{p}(\Omega) .
$$

If $K$ is a convex subset of $X$ and $u \in K$, then $\alpha \in \partial^{-} I_{K}(u)$ if and only if

$$
\alpha(v-u) \leq 0 \quad \forall v \in K
$$

REMARK 1.3. Notice that, if $\Phi$ and $\Gamma$ are two functions in $X$ with values in $\mathbb{R} \cup\{\infty\}$ and if $u \in D(\Phi+\Gamma)$, then

$$
\partial^{-} \Phi(u)+\partial^{-} \Gamma(u) \subseteq \partial^{-}(\Phi+\Gamma)(u) .
$$

Simple examples show that, in general, the opposite inclusion does not hold. However, if the function $\Phi$ is differentiable at $u$, then

$$
\left\{\Phi^{\prime}(u)\right\}+\partial^{-} \Gamma(u)=\partial^{-}(\Phi+\Gamma)(u) .
$$

Definition 1.4. Let $K$ be a subset of $H_{0}^{1,2}(\Omega) \cap L^{p}(\Omega)$ and $u \in K \cap V$. We say that $K$ and $V$ are nontangent at $u$ if

$$
\partial^{-} I_{K}(u) \cap \partial^{-} I_{V}(u)=\{0\} .
$$


Proposition 1.5. Let $K$ be a subset of $H_{0}^{1,2}(\Omega) \cap L^{p}(\Omega)$ and $u \in K \cap V$. If $K$ and $V$ are nontangent at $u$, then

$$
\partial^{-}\left(I_{K}+I_{V}\right)(u)=\partial^{-} I_{K}(u)+\partial^{-} I_{V}(u) .
$$

For the proof it suffices to argue as in [13] and [14].

All the domains $\Omega \subset \mathbb{R}^{n}$ we shall consider in this paper have radial symmetry with respect to the $x_{n}$-axis; therefore it is natural to look for solutions of $P(\Omega, p)$ in the space of functions having the same symmetry.

DEFinition 1.6. We say that $\Omega$ has radial symmetry with respect to the $x_{n}$-axis if the following property holds:

$$
x \in \Omega \Leftrightarrow\left(0, \ldots, 0, \varrho(x), x_{n}\right) \in \Omega \text { where } \varrho(x)=\left(\sum_{i=1}^{n-1} x_{i}^{2}\right)^{1 / 2} .
$$

Analogously, we say that a function $u$, defined in a domain $\Omega$ having radial symmetry with respect to $x_{n}$-axis, has the same symmetry if

$$
u(x)=u\left(0, \ldots, 0, \varrho(x), x_{n}\right) \quad \forall x \in \Omega .
$$

If $\Omega$ has radial symmetry, we shall denote by $H_{S}(\Omega)$ the subspace of $H_{0}^{1,2}(\Omega)$ consisting of the radial functions.

It is well known that every critical point for the functional $f$ constrained on $H_{S}(\Omega) \cap L^{p}(\Omega)$ is a critical point for $f$ in $H_{0}^{1,2}(\Omega) \cap L^{p}(\Omega)$.

REMARK 1.7. Notice that, if $\Omega$ is a bounded domain having radial symmetry with respect to the $x_{n}$-axis and moreover

$$
\inf \left\{\sum_{i=1}^{n-1} x_{i}^{2}: x=\left(x_{1}, \ldots, x_{n}\right) \in \Omega\right\}>0,
$$

then the space $H_{S}(\Omega)$ is isomorphic to $H_{0}^{1,2}(\widetilde{\Omega})$, where $\widetilde{\Omega}=\left\{(\varrho, t) \in \mathbb{R}^{2}\right.$ : $(0, \ldots, 0, \varrho, t) \in \Omega\}\left(\widetilde{\Omega}\right.$ is a bounded domain in $\left.\mathbb{R}^{2}\right)$. Consequently, $H_{S}(\Omega)$ is compactly embedded in $L^{q}(\Omega)$ for all $q \geq 1$.

\section{Nodal solutions in contractible domains}

In this section we obtain for all $p>2 n /(n-2)$ a bounded contractible domain $\Omega$ (having radial symmetry with respect to the $x_{n}$-axis) such that problem $P(\Omega, p)$ has nodal solutions (see Theorem 2.2).

Notations 2.1. Set $B(x, \varrho)=\left\{y \in \mathbb{R}^{n}:|y-x|<\varrho\right\}$ for $x \in \mathbb{R}^{n}$ and for $\varrho>0$. For all positive integer $h$, let

$$
T_{h}=\left\{x=\left(x_{1}, \ldots, x_{n}\right) \in \mathbb{R}^{n}: \sum_{i=1}^{n-1} x_{i}^{2}<1,0<x_{n}<h+1\right\} ;
$$


for $i=1, \ldots, h$ put $c_{i}=(0, \ldots, 0, i) \in \mathbb{R}^{n}$; given $\sigma_{1}, \ldots, \sigma_{h}$ with $0<\sigma_{i}<1 / 2$ for $i=1, \ldots, h$, set

$D_{h}=T_{h} \backslash \bigcup_{i=1}^{h} \overline{B\left(c_{i}, \sigma_{i}\right)}, \quad \chi_{\varepsilon}=\left\{x \in \mathbb{R}^{n}: \sum_{i=1}^{n-1} x_{i}^{2} \leq \varepsilon^{2}, \quad x_{n} \geq 1\right\}, \quad \Omega_{\varepsilon}^{h}=D_{h} \backslash \chi_{\varepsilon}$.

For all $\varepsilon>0$, every function $u$ of $H_{0}^{1,2}\left(\Omega_{\varepsilon}^{h}\right)$ is understood to be extended in $D_{h}$ by zero.

Moreover, put

$$
\|u\|_{q}=\left(\int_{D_{h}}|u|^{q} d x\right)^{1 / q} \forall u \in L^{q}\left(D_{h}\right) \quad(q \geq 1) .
$$

Notice that all the domains $T_{h}, D_{h}$ and $\Omega_{\varepsilon}^{h}$ have radial symmetry with respect to the $x_{n}$-axis; moreover, $\Omega_{\varepsilon}^{h}$ is a bounded contractible domain for all $\varepsilon>0$.

Set

$$
F_{i}=\left\{x=\left(x_{1}, \ldots, x_{n}\right) \in \mathbb{R}^{n}: i \leq x_{n} \leq i+1\right\} \quad \forall i=1, \ldots, h ;
$$

for every pair $(r, s)$, with $r, s=1, \ldots, h$, let $K^{r, s}$ be the set defined by

$$
\begin{array}{r}
K^{r, s}=\left\{u \in H_{S}\left(D_{h}\right): u^{ \pm} \not \equiv 0, u^{+}(x) /\left\|u^{+}\right\|_{p} \leq 1 \forall x \in D_{h} \backslash F_{r},\right. \\
\left.u^{-}(x) /\left\|u^{-}\right\|_{p} \leq 1 \forall x \in D_{h} \backslash F_{s}\right\} .
\end{array}
$$

Theorem 2.2. Let $p>2 n /(n-2)$. For all $\varepsilon>0$ and $h \in \mathbb{N}$ let $\Omega_{\varepsilon}^{h}$ be the domain defined above. Then there exists $\bar{\varepsilon}>0$ such that, for all $\varepsilon \in$ ] $0, \bar{\varepsilon}\left[\right.$, problem $P\left(\Omega_{\varepsilon}^{h}, p\right)$ has at least $h^{2}$ distinct solutions $u_{r, s, \varepsilon}(r, s=1, \ldots, h)$. Moreover, for every pair $(r, s)$, the solution $u_{r, s, \varepsilon}$ minimizes the functional $f$ in $H_{s}\left(\Omega_{\varepsilon}^{h}\right) \cap M \cap K^{r, s}$ (see (1.1), (1.2) and Notations 2.1). Furthermore, we have

$$
\lim _{\varepsilon \rightarrow 0^{+}} f\left(u_{r, s, \varepsilon}\right)=0 .
$$

The proof is based on the following lemmas.

Lemma 2.3. There exists $\bar{\varepsilon}>0$ such that, for all $\varepsilon \in] 0, \bar{\varepsilon}[$, the minimum

$$
\min \left\{f(u): u \in H_{S}\left(\Omega_{\varepsilon}^{h}\right) \cap M \cap K^{r, s}\right\}
$$

is achieved. Moreover,

$$
\lim _{\varepsilon \rightarrow 0^{+}} \min \left\{f(u): u \in H_{S}\left(\Omega_{\varepsilon}^{h}\right) \cap M \cap K^{r, s}\right\}=0 .
$$

Proof. Fix $r$ and $s$ in $\{1, \ldots, h\}$. First remark that

$$
\lim _{\varepsilon \rightarrow 0^{+}} \inf \left\{f(u): u \in H_{S}\left(\Omega_{\varepsilon}^{h}\right) \cap M \cap K^{r, s}\right\}=0 .
$$


In fact, choose $z \in H_{S}\left(\Omega_{0}\right)$ with

$$
\Omega_{0}=\left\{x=\left(x_{1}, \ldots, x_{n}\right) \in \mathbb{R}^{n}:|x|<2, \sum_{i=1}^{n-1} x_{i}^{2}>1\right\}
$$

such that $z \geq 0$ in $\Omega_{0}$ and $\int_{\Omega_{0}} z^{p} d x=1$; for all $\varepsilon>0$ and $\bar{x} \in \mathbb{R}^{n}$ set $z_{\varepsilon, \bar{x}}(x)=$ $z\left((x-\bar{x}) / \varepsilon\right.$ ) (we understand that $z$ is extended by zero outside $\Omega_{0}$ ); fix $\bar{x}^{(r)}=$ $\left(0, \ldots, 0, \bar{x}_{n}^{(r)}\right)$ and $\bar{x}^{(s)}=\left(0, \ldots, 0, \bar{x}_{n}^{(s)}\right)$ such that $\bar{x}^{(r)} \neq \bar{x}^{(s)}$ and (see Notations 2.1)

$$
r+\sigma_{r}<\bar{x}_{n}^{(r)}<r+1-\sigma_{(r+1)}, \quad s+\sigma_{s}<\bar{x}_{n}^{(s)}<s+1-\sigma_{(s+1)}
$$

if we set $\zeta_{\varepsilon}=\left(\left\|D z_{\varepsilon}\right\|_{2}^{2} /\left\|z_{\varepsilon}\right\|_{p}^{p}\right)^{1 /(p-2)}\left(z_{\varepsilon, \bar{x}^{(r)}}-z_{\left.\varepsilon, \bar{x}^{(s)}\right)}\right.$, it is easy to verify that $\zeta_{\varepsilon} \in H_{S}\left(\Omega_{\varepsilon}^{h}\right) \cap M \cap K^{r, s}$ for $\varepsilon>0$ small enough and that $\lim _{\varepsilon \rightarrow 0} f\left(\zeta_{\varepsilon}\right)=0$.

Now set (see Notations 2.1)

$$
\lambda=\min \left\{\int_{D_{h} \cap F_{0}}|D u|^{2} d x: u \in H_{0}^{1,2}\left(D_{h}\right), \int_{D_{h} \cap F_{0}} u^{2} d x=1\right\} .
$$

Notice that, if $u \in H_{0}^{1,2}\left(\Omega_{\varepsilon}^{h}\right)$ satisfies

$$
0<\sup \left\{u(x): x \in \Omega_{\varepsilon}^{h} \cap F_{0}\right\}<\lambda^{1 /(p-2)},
$$

then

$$
\int_{\Omega_{\varepsilon}^{h} \cap F_{0}}|u|^{p} d x<\lambda \int_{\Omega_{\varepsilon}^{h} \cap F_{0}} u^{2} d x \leq \int_{\Omega_{\varepsilon}^{h} \cap F_{0}}|D u|^{2} d x,
$$

because $|u(x)|^{p}<\lambda u^{2}(x)$ if $0<|u(x)|<\lambda^{1 /(p-2)}$.

Let $\left(u_{\varepsilon, i}\right)_{i}$ be a minimizing sequence for $f$ on $H_{S}\left(\Omega_{\varepsilon}^{h}\right) \cap M \cap K^{r, s}$. Since (2.1) holds, we can assume that, for $\varepsilon>0$ small enough,

$$
\left\|D u_{\varepsilon, i}^{ \pm}\right\|_{2}^{2}=\left\|u_{\varepsilon, i}^{ \pm}\right\|_{p}^{p}<\lambda^{p /(p-2)} \quad \forall i \in \mathbb{N} ;
$$

in particular, since $u_{\varepsilon, i} \in K^{r, s}$ for all $i \in \mathbb{N}$, we have

$$
\sup \left\{u_{\varepsilon, i}^{ \pm}(x): x \in \Omega_{\varepsilon}^{h} \cap F_{0}\right\}<\lambda^{1 /(p-2)} \quad \forall i \in \mathbb{N} .
$$

Let us prove that, up to a subsequence, $\left(u_{\varepsilon, i}\right)_{i}$ converges in $H_{0}^{1,2}\left(\Omega_{\varepsilon}^{h}\right)$ to a function $u_{\varepsilon}$ which yields the minimum of $f$ on $H_{S}\left(\Omega_{\varepsilon}^{h}\right) \cap M \cap K^{r, s}$. In fact, since the sequence $\left(u_{\varepsilon, i}\right)_{i}$ is bounded in $H_{0}^{1,2}\left(\Omega_{\varepsilon}^{h}\right)$, up to a subsequence we have $\left(u_{\varepsilon, i}\right)_{i} \rightarrow u_{\varepsilon}$ and $\left(u_{\varepsilon, i}^{ \pm}\right)_{i} \rightarrow u_{\varepsilon}^{ \pm}$weakly in $H_{0}^{1,2}\left(\Omega_{\varepsilon}^{h}\right)$, in $L^{2}\left(\Omega_{\varepsilon}^{h}\right)$ and almost everywhere in $\Omega_{\varepsilon}^{h}$, for a suitable $u_{\varepsilon} \in H_{S}\left(\Omega_{\varepsilon}^{h}\right)$.

Then it suffices to prove that:

(a) $\lim _{i \rightarrow \infty}\left\|u_{\varepsilon, i}^{ \pm}\right\|_{p}=\left\|u_{\varepsilon}^{ \pm}\right\|_{p}>0$

(b) $\lim _{i \rightarrow \infty}\left\|D u_{\varepsilon, i}^{ \pm}\right\|_{2}=\left\|D u_{\varepsilon}^{ \pm}\right\|_{2}$, 
because these properties imply $u_{\varepsilon} \in H_{S}\left(\Omega_{\varepsilon}^{h}\right) \cap M \cap K^{r, s}$ and

$$
f\left(u_{\varepsilon}\right)=\lim _{i \rightarrow \infty} f\left(u_{\varepsilon, i}\right)=\inf \left\{f(u): u \in H_{S}\left(\Omega_{\varepsilon}^{h}\right) \cap M \cap K^{r, s}\right\} .
$$

To prove (a), we remark that, since

$$
\inf \left\{\sum_{i=1}^{n-1} x_{i}^{2}: x \in \Omega_{\varepsilon}^{h} \backslash F_{0}\right\}>0,
$$

the subspace of $H^{1,2}\left(\Omega_{\varepsilon}^{h} \backslash F_{0}\right)$ consisting of the radial functions vanishing on $\partial \Omega_{\varepsilon}^{h}$ (in the sense of $H^{1,2}$ ), endowed with the equivalent norm

$$
\|u\|=\left(\int_{\Omega_{\varepsilon}^{h} \backslash F_{0}}|D u|^{2} d x\right)^{1 / 2},
$$

is compactly embedded in $L^{p}\left(\Omega_{\varepsilon}^{h} \backslash F_{0}\right)$ for all $p \geq 1$. Therefore we have

$$
\lim _{i \rightarrow \infty} \int_{\Omega_{\varepsilon}^{h} \backslash F_{0}}\left(u_{\varepsilon, i}^{ \pm}\right)^{p} d x=\int_{\Omega_{\varepsilon}^{h} \backslash F_{0}}\left(u_{\varepsilon}^{ \pm}\right)^{p} d x .
$$

Moreover,

$$
\lim _{i \rightarrow \infty} \int_{\Omega_{\varepsilon}^{h} \cap F_{0}}\left(u_{\varepsilon, i}^{ \pm}\right)^{p} d x=\int_{\Omega_{\varepsilon}^{h} \cap F_{0}}\left(u_{\varepsilon}^{ \pm}\right)^{p} d x
$$

by the Lebesgue theorem, because of (2.3), and so $\lim _{i \rightarrow \infty}\left\|u_{\varepsilon, i}^{ \pm}\right\|_{p}=\left\|u_{\varepsilon}^{ \pm}\right\|_{p}$.

Let us prove that $\left\|u_{\varepsilon}^{+}\right\|_{p}>0:(2.4)$ implies that there exists a constant $c_{\varepsilon}>0$ such that

$$
c_{\varepsilon}\left(\int_{\Omega_{\varepsilon}^{h} \backslash F_{0}}|u|^{p} d x\right)^{1 / p} \leq\left(\int_{\Omega_{\varepsilon}^{h} \backslash F_{0}}|D u|^{2} d x\right)^{1 / 2} \forall u \in H_{S}\left(\Omega_{\varepsilon}^{h}\right) .
$$

Then, if $\sup \left\{u_{\varepsilon, i}^{+}(x): x \in \Omega_{\varepsilon}^{h} \cap F_{0}\right\}=0$, we have (since $u_{\varepsilon, i} \in M$ )

$$
\left\|u_{\varepsilon, i}^{+}\right\|_{p}^{p}=\left\|D u_{\varepsilon, i}^{+}\right\|_{2}^{2} \geq c_{\varepsilon}^{2}\left\|u_{\varepsilon, i}^{+}\right\|_{p}^{2}
$$

and so (as $\left.\left\|u_{\varepsilon, i}^{+}\right\|_{p} \neq 0\right)$

$$
\left\|u_{\varepsilon, i}^{+}\right\|_{p} \geq c_{\varepsilon}^{2 /(p-2)} .
$$

On the contrary, if

$$
\sup \left\{u_{\varepsilon, i}^{+}(x): x \in \Omega_{\varepsilon}^{h} \cap F_{0}\right\}>0,
$$

then from (2.3) and (2.2) it follows that

$$
\int_{\Omega_{\varepsilon}^{h} \cap F_{0}}\left|u_{\varepsilon, i}^{+}\right|^{p} d x<\int_{\Omega_{\varepsilon}^{h} \cap F_{0}}\left|D u_{\varepsilon, i}^{+}\right|^{2} d x,
$$

which implies, since $u_{\varepsilon, i} \in M$,

$$
\int_{\Omega_{\varepsilon}^{h} \backslash F_{0}}\left|u_{\varepsilon, i}^{+}\right|^{p} d x>\int_{\Omega_{\varepsilon}^{h} \backslash F_{0}}\left|D u_{\varepsilon, i}^{+}\right|^{2} d x .
$$


Consequently, we have

$$
\left(\int_{\Omega_{\varepsilon}^{h} \backslash F_{0}}\left|u_{\varepsilon, i}^{+}\right|^{p} d x\right)^{1 / p}>c_{\varepsilon}^{2 /(p-2)},
$$

which implies (2.5) in this case too; so

$$
\left\|u_{\varepsilon}^{+}\right\|_{p}=\lim _{i \rightarrow \infty}\left\|u_{\varepsilon, i}^{+}\right\|_{p} \geq c_{\varepsilon}^{2 /(p-2)}>0 .
$$

In an analogous way one can prove that $\left\|u_{\varepsilon}^{-}\right\|_{p} \geq c_{\varepsilon}^{2 /(p-2)}$.

Let us prove (b): since $u_{\varepsilon, i} \in M$ for all $i \in \mathbb{N}$, we have $\lim _{i \rightarrow \infty}\left\|D u_{\varepsilon, i}^{ \pm}\right\|_{2}^{2}=$ $\lim _{i \rightarrow \infty}\left\|u_{\varepsilon, i}^{ \pm}\right\|_{p}^{p}=\left\|u_{\varepsilon}^{ \pm}\right\|_{p}^{p}$. Thus we have to prove that $\left\|D u_{\varepsilon}^{ \pm}\right\|_{2}^{2}=\left\|u_{\varepsilon}^{ \pm}\right\|_{p}^{p}$.

Notice that $0<\left\|D u_{\varepsilon}^{ \pm}\right\|_{2}^{2} \leq\left\|u_{\varepsilon}^{ \pm}\right\|_{p}^{p}$; so, if we set $t_{\varepsilon}^{ \pm}=\left(\left\|D u_{\varepsilon}^{ \pm}\right\|_{2}^{2} /\left\|u_{\varepsilon}^{ \pm}\right\|_{p}^{p}\right)^{1 /(p-2)}$, we have $0<t_{\varepsilon}^{ \pm} \leq 1$.

Consequently, the function

$$
\widetilde{u}_{\varepsilon}=t_{\varepsilon}^{+} u_{\varepsilon}^{+}-t_{\varepsilon}^{-} u_{\varepsilon}^{-}
$$

lies in $H_{S}\left(\Omega_{\varepsilon}^{h}\right) \cap M \cap K^{r, s}$ and so

$$
f\left(\widetilde{u}_{\varepsilon}\right) \geq \inf \left\{f(u): u \in H_{S}\left(\Omega_{\varepsilon}^{h}\right) \cap M \cap K^{r, s}\right\} .
$$

On the other hand, we have $f(u)=(1 / 2-1 / p)\|D u\|_{2}^{2}$ for all $u \in M$. Therefore

$$
\begin{aligned}
f\left(\widetilde{u}_{\varepsilon}\right) & =(1 / 2-1 / p)\left\|D \widetilde{u}_{\varepsilon}\right\|_{2}^{2} \\
& =(1 / 2-1 / p)\left[\left(t_{\varepsilon}^{+}\right)^{2}\left\|D u_{\varepsilon}^{+}\right\|_{2}^{2}+\left(t_{\varepsilon}^{-}\right)^{2}\left\|D u_{\varepsilon}^{-}\right\|_{2}^{2}\right] \\
& \leq(1 / 2-1 / p)\left\|D u_{\varepsilon}\right\|_{2}^{2} \leq \lim _{i \rightarrow \infty}(1 / 2-1 / p)\left\|D u_{\varepsilon, i}\right\|_{2}^{2} \\
& =\lim _{i \rightarrow \infty} f\left(u_{\varepsilon, i}\right)=\inf \left\{f(u): u \in H_{S}\left(\Omega_{\varepsilon}^{h}\right) \cap M \cap K^{r, s}\right\} .
\end{aligned}
$$

Taking into account (2.6), we have in particular

$$
\left[1-\left(t_{\varepsilon}^{+}\right)^{2}\right]\left\|D u_{\varepsilon}^{+}\right\|_{2}^{2}+\left[1-\left(t_{\varepsilon}^{-}\right)^{2}\right]\left\|D u_{\varepsilon}^{-}\right\|_{2}^{2}=0
$$

with $1-\left(t_{\varepsilon}^{ \pm}\right)^{2} \geq 0$ and $\left\|D u_{\varepsilon}^{ \pm}\right\|_{2}^{2}>0$. Therefore we must have $t_{\varepsilon}^{ \pm}=1$, that is, $\left\|D u_{\varepsilon}^{ \pm}\right\|_{2}^{2}=\left\|u_{\varepsilon}^{ \pm}\right\|_{p}^{p}$.

REMARK 2.4. It is easy to verify that, on the contrary, the minimum of $f$ on $H_{S}\left(\Omega_{\varepsilon}^{h}\right) \cap M$ does not exist and that $\inf \left\{f(u): u \in H_{S}\left(\Omega_{\varepsilon}^{h}\right) \cap M\right\}=0$ for all $\varepsilon>0$ (see also Remark 3.4).

Lemma 2.5. For all $\varepsilon \in] 0, \bar{\varepsilon}\left[\left(\right.\right.$ see Lemma 2.3), let $u_{r, s, \varepsilon} \in H_{S}\left(\Omega_{\varepsilon}^{h}\right)$ be a minimum point for $f$ on $H_{S}\left(\Omega_{\varepsilon}^{h}\right) \cap M \cap K^{r, s}$. Then the function $u_{r, s, \varepsilon}^{+}$minimizes the functional $g(u)=\|D u\|_{2}^{2}$ in the set

$$
\begin{aligned}
& \left\{u \in H_{S}\left(\Omega_{\varepsilon}^{h}\right): u \geq 0 \text { in } \Omega_{\varepsilon}^{h}, \quad \int_{\Omega_{\varepsilon}^{h}} u u_{r, s, \varepsilon}^{-} d x=0,\right. \\
& \left.\qquad\|u\|_{p}=\left\|u_{r, s, \varepsilon}^{+}\right\|_{p}, u(x) \leq\left\|u_{r, s, \varepsilon}^{+}\right\|_{p} \forall x \in \Omega_{\varepsilon}^{h} \backslash F_{r}\right\} .
\end{aligned}
$$

An analogous property holds for $u_{r, s, \varepsilon}^{-}$. 
Proof. Suppose, by contradiction, that there exists $\bar{u} \in H_{S}\left(\Omega_{\varepsilon}^{h}\right)$ such that $\bar{u} \geq 0$ in $\Omega_{\varepsilon}^{h}$,

$$
\begin{gathered}
\int_{\Omega_{\varepsilon}^{h}} \bar{u} u_{r, s, \varepsilon}^{-} d x=0, \quad\|\bar{u}\|_{p}=\left\|u_{r, s, \varepsilon}^{+}\right\|_{p}, \\
\bar{u}(x) \leq\left\|u_{r, s, \varepsilon}^{+}\right\|_{p} \quad \forall x \in \Omega_{\varepsilon}^{h} \backslash F_{r}, \quad\|D \bar{u}\|_{2}<\left\|D u_{r, s, \varepsilon}^{+}\right\|_{2} .
\end{gathered}
$$

If we set $\bar{t}=\left(\|D \bar{u}\|_{2}^{2} /\|\bar{u}\|_{p}^{p}\right)^{1 /(p-2)}$ and $\widetilde{u}=\bar{t} \bar{u}-u_{r, s, \varepsilon}^{-}$, we have $0<\bar{t}<1$ and $\widetilde{u} \in H_{S}\left(\Omega_{\varepsilon}^{h}\right) \cap M \cap K^{r, s}$. It follows that

$$
\begin{aligned}
f(\widetilde{u}) & =(1 / 2-1 / p)\|D \widetilde{u}\|_{2}^{2}=(1 / 2-1 / p)\left[\bar{t}\|D \bar{u}\|_{2}^{2}+\left\|D u_{r, s, \varepsilon}^{-}\right\|_{2}^{2}\right] \\
& <(1 / 2-1 / p)\left[\left\|D u_{r, s, \varepsilon}^{+}\right\|_{2}^{2}+\left\|D u_{r, s, \varepsilon}^{-}\right\|_{2}^{2}\right]=f\left(u_{r, s, \varepsilon}\right),
\end{aligned}
$$

which is a contradiction since $u_{r, s, \varepsilon}$ minimizes $f$ in $H_{S}\left(\Omega_{\varepsilon}^{h}\right) \cap M \cap K^{r, s}$.

Lemma 2.6. For all $\varepsilon \in] 0, \bar{\varepsilon}\left[\right.$ (see Lemma 2.3), let $u_{r, s, \varepsilon}$ be a minimum point for $f$ on $H_{S}\left(\Omega_{\varepsilon}^{h}\right) \cap M \cap K^{r, s}$. Then there exists $\bar{\varepsilon}_{1}>0$ such that:

(a) For all $\varepsilon \in] 0, \bar{\varepsilon}_{1}[$ the convex set

$$
K_{\varepsilon}^{r}=\left\{u \in H_{S}\left(\Omega_{\varepsilon}^{h}\right): \int_{\Omega_{\varepsilon}^{h}}|u| u_{r, s, \varepsilon}^{-} d x=0 ;|u(x)| \leq 1 \forall x \in \Omega_{\varepsilon}^{h} \backslash F_{r}\right\}
$$

and the manifold

$$
V_{\varepsilon}=\left\{u \in H_{S}\left(\Omega_{\varepsilon}^{h}\right): \int_{\Omega_{\varepsilon}^{h}}|u|^{p} d x=1\right\}
$$

are nontangent at $u_{r, s, \varepsilon}^{+} /\left\|u_{r, s, \varepsilon}^{+}\right\|_{p}$.

(b) For all $\varepsilon \in] 0, \bar{\varepsilon}_{1}\left[\right.$ there exists $\lambda_{\varepsilon} \in \mathbb{R}$ such that, for all $v \in K_{\varepsilon}^{r}$,

$\int_{\Omega_{\varepsilon}^{h}} \frac{D u_{r, s, \varepsilon}^{+}}{\left\|u_{r, s, \varepsilon}^{+}\right\|_{p}} D\left(v-\frac{u_{r, s, \varepsilon}^{+}}{\left\|u_{r, s, \varepsilon}^{+}\right\|_{p}}\right) d x-\lambda_{\varepsilon} \int_{\Omega_{\varepsilon}^{h}} \frac{\left(u_{r, s, \varepsilon}^{+}\right)^{p-1}}{\left\|u_{r, s, \varepsilon}^{+}\right\|_{p}^{p-1}}\left(v-\frac{u_{r, s, \varepsilon}^{+}}{\left\|u_{r, s, \varepsilon}^{+}\right\|_{p}}\right) d x \geq 0$.

Moreover, $\lim _{\varepsilon \rightarrow 0^{+}} \lambda_{\varepsilon}=0$.

(c) For all nonnegative, radially symmetric functions $w \in C_{0}^{\infty}\left(\Omega_{\varepsilon}^{h}\right)$ we have

$$
\int_{\Omega_{\varepsilon}^{h}} \frac{D u_{r, s, \varepsilon}^{+}}{\left\|u_{r, s, \varepsilon}^{+}\right\|_{p}} D w d x-\lambda_{\varepsilon} \int_{\Omega_{\varepsilon}^{h}} \frac{\left(u_{r, s, \varepsilon}^{+}\right)^{p-1}}{\left\|u_{r, s, \varepsilon}^{+}\right\|_{p}^{p-1}} w d x \leq 0 .
$$

(d) $\lim _{\varepsilon \rightarrow 0^{+}} \sup \left\{u_{r, s, \varepsilon}^{+}(x) /\left\|u_{r, s, \varepsilon}^{+}\right\|_{p}: x \in \Omega_{\varepsilon}^{h} \backslash F_{r}\right\}=0$.

Analogous properties hold for the function $u_{r, s, \varepsilon}^{-}$.

Proof. Notice that, since $u_{r, s, \varepsilon}^{+}$minimizes the functional $g(u)=\|D u\|_{2}^{2}$ in the set

$$
\begin{array}{r}
\left\{u \in H_{S}\left(\Omega_{\varepsilon}^{h}\right): \int_{\Omega_{\varepsilon}^{h}}|u| u_{r, s, \varepsilon}^{-} d x=0,\|u\|_{p}=\left\|u_{r, s, \varepsilon}^{+}\right\|_{p},\right. \\
\left.|u(x)| \leq\left\|u_{r, s, \varepsilon}^{+}\right\|_{p} \forall x \in \Omega_{\varepsilon}^{h} \backslash F_{r}\right\},
\end{array}
$$


the function $u_{r, s, \varepsilon}^{+} /\left\|u_{r, s, \varepsilon}^{+}\right\|_{p}$ minimizes $g$ in $K_{\varepsilon}^{r} \cap V_{\varepsilon}$; moreover,

$$
\lim _{\varepsilon \rightarrow 0^{+}}\left\|D u_{r, s, \varepsilon}^{+}\right\|_{2} /\left\|u_{r, s, \varepsilon}^{+}\right\|_{p}=0 .
$$

Using these properties, the proof can be concluded arguing as in [35] (see Lemmas $2.5-2.8$ of $[35])$.

Definition 2.7. For all $\varepsilon \in] 0, \bar{\varepsilon}_{1}$ [ (see Lemma 2.6), let $u_{r, s, \varepsilon}$ be a function minimizing $f$ on $H_{S}\left(\Omega_{\varepsilon}^{h}\right) \cap M \cap K^{r, s}$. For all $\left.\delta \in\right] 0, \pi / 4\left[\right.$ and $\left.\varrho_{0} \in\right] 0,1[$ set $Q_{\varrho_{0}, \delta}=\left[\varrho_{0}, 1\right] \times[\delta, \pi / 2-\delta]$ and define $\sigma: Q_{\varrho_{0}, \delta} \rightarrow H_{S}\left(\Omega_{\varepsilon}^{h}\right) \cap K^{r, s}$ by

$$
\sigma(\varrho, \vartheta)=\frac{u_{r, s, \varepsilon}^{+}}{\left\|u_{r, s, \varepsilon}^{+}\right\|_{p}} \varrho \cos \vartheta-\frac{u_{r, s, \varepsilon}^{-}}{\left\|u_{r, s, \varepsilon}^{-}\right\|_{p}} \varrho \sin \vartheta
$$

(notice that $\sigma(\varrho, \vartheta) \neq 0$ for $\left.(\varrho, \vartheta) \in Q_{\varrho_{0}, \delta}\right)$.

Proof of Theorem 2.2. We shall only prove that, for $r, s=1, \ldots, h$ and for $\varepsilon>0$ small enough, every function $u_{r, s, \varepsilon}$ which minimizes $f$ on $H_{S}\left(\Omega_{\varepsilon}^{h}\right) \cap$ $M \cap K^{r, s}$ is a solution of problem $P\left(\Omega_{\varepsilon}^{h}, p\right)$; the fact that different solutions $u_{r, s, \varepsilon}$ correspond to different pairs $(r, s)$ will follow from the behaviour of the functions $u_{r, s, \varepsilon}$, stated in Section 3 (see Proposition 3.2).

Since $\lim _{\varepsilon \rightarrow 0^{+}}\left\|D u_{r, s, \varepsilon}\right\|_{2} /\left\|u_{r, s, \varepsilon}\right\|_{p}=0$ (see Lemma 2.3), and (see Lemma 2.6)

$$
\begin{aligned}
& \lim _{\varepsilon \rightarrow 0^{+}} \sup \left\{\frac{u_{r, s, \varepsilon}^{+}(x)}{\left\|u_{r, s, \varepsilon}^{+}\right\|_{p}}: x \in \Omega_{\varepsilon}^{h} \backslash F_{r}\right\}=0, \\
& \lim _{\varepsilon \rightarrow 0^{+}} \sup \left\{\frac{u_{r, s, \varepsilon}^{-}(x)}{\left\|u_{r, s, \varepsilon}^{-}\right\|_{p}}: x \in \Omega_{\varepsilon}^{h} \backslash F_{s}\right\}=0,
\end{aligned}
$$

for $\varepsilon>0$ small enough we have

$$
\begin{gathered}
\left\{\begin{array}{l}
\sup \left\{u_{r, s, \varepsilon}^{+}(x) /\left\|u_{r, s, \varepsilon}^{+}\right\|_{p}: x \in \Omega_{\varepsilon}^{h} \backslash F_{r}\right\}<1, \\
\sup \left\{u_{r, s, \varepsilon}^{-}(x) /\left\|u_{r, s, \varepsilon}^{-}\right\|_{p}: x \in \Omega_{\varepsilon}^{h} \backslash F_{s}\right\}<1 ;
\end{array}\right. \\
\min \left\{(\cos \vartheta)^{p-2} \frac{\left\|u_{r, s, \varepsilon}^{+}\right\|_{p}^{2}}{\left\|D u_{r, s, \varepsilon}^{+}\right\|_{2}^{2}}+(\sin \vartheta)^{p-2} \frac{\left\|u_{r, s, \varepsilon}^{-}\right\|_{p}^{2}}{\left\|D u_{r, s, \varepsilon}^{-}\right\|_{2}^{2}}: \vartheta \in[0, \pi / 2]\right\} \geq 2 .
\end{gathered}
$$

Fix $\varepsilon>0$ in such a way that properties (2.8) and (2.9) hold and $\left(\left\|u_{r, s, \varepsilon}^{+}\right\|_{p}^{2}+\right.$ $\left.\left\|u_{r, s, \varepsilon}^{-}\right\|_{p}^{2}\right)^{1 / 2}<1$; choose $\varrho_{0}>0$ small enough that

$$
\begin{gathered}
\varrho_{0}<\left(\left\|u_{r, s, \varepsilon}^{+}\right\|_{p}^{2}+\left\|u_{r, s, \varepsilon}^{-}\right\|_{p}^{2}\right)^{1 / 2} \\
\max \left\{\left(\varrho_{0} \cos \vartheta\right)^{p-2} \frac{\left\|u_{r, s, \varepsilon}^{+}\right\|_{p}^{2}}{\left\|D u_{r, s, \varepsilon}^{+}\right\|_{2}^{2}}+\left(\varrho_{0} \sin \vartheta\right)^{p-2} \frac{\left\|u_{r, s, \varepsilon}^{-}\right\|_{p}^{2}}{\left\|D u_{r, s, \varepsilon}^{-}\right\|_{2}^{2}}:\right. \\
\vartheta \in[0, \pi / 2]\} \leq 2 .
\end{gathered}
$$


Then choose $\delta \in] 0, \pi / 4\left[\right.$ in such a way that the pair $\left(\varrho_{\varepsilon}, \vartheta_{\varepsilon}\right)$ satisfying $\sigma\left(\varrho_{\varepsilon}, \vartheta_{\varepsilon}\right)=$ $u_{r, s, \varepsilon}$ lies in the interior of $Q_{\varrho_{0}, \delta}$ and, moreover,

$$
\begin{gathered}
\min \left\{(\varrho \cos \delta)^{p-2} \frac{\left\|u_{r, s, \varepsilon}^{+}\right\|_{p}^{2}}{\left\|D u_{r, s, \varepsilon}^{+}\right\|_{2}^{2}}: \varrho \in\left[\varrho_{0}, 1\right]\right\} \geq(\sin \delta)^{p-2} \frac{\left\|u_{r, s, \varepsilon}^{-}\right\|_{p}^{2}}{\left\|D u_{r, s, \varepsilon}^{-}\right\|_{2}^{2}}, \\
(\sin \delta)^{p-2} \frac{\left\|u_{r, s, \varepsilon}^{+}\right\|_{p}^{2}}{\left\|D u_{r, s, \varepsilon}^{+}\right\|_{2}^{2}} \leq \min \left\{(\varrho \cos \delta)^{p-2} \frac{\left\|u_{r, s, \varepsilon}^{-}\right\|_{p}^{2}}{\left\|D u_{r, s, \varepsilon}^{-}\right\|_{2}^{2}}: \varrho \in\left[\varrho_{0}, 1\right]\right\} .
\end{gathered}
$$

In order to show that $u_{r, s, \varepsilon}$ is a critical point for $f$, it suffices to prove that

$$
f^{\prime}\left(u_{r, s, \varepsilon}\right)[w]=0 \quad \forall w \in C_{0}^{\infty}\left(\Omega_{\varepsilon}^{h}\right) \cap H_{S}\left(\Omega_{\varepsilon}^{h}\right) .
$$

Suppose, by contradiction, that there exists $\bar{w} \in C_{0}^{\infty}\left(\Omega_{\varepsilon}^{h}\right) \cap H_{S}\left(\Omega_{\varepsilon}^{h}\right)$ such that $f^{\prime}\left(u_{r, s, \varepsilon}\right)[\bar{w}]<0$. Then there exists a neighbourhood $I\left(u_{r, s, \varepsilon}\right)$ of $u_{r, s, \varepsilon}$ in $H_{S}\left(\Omega_{\varepsilon}^{h}\right)$ $\cap L^{p}\left(\Omega_{\varepsilon}^{h}\right)$ such that $f^{\prime}(u)[\bar{w}]<0$ for $u \in I\left(u_{r, s, \varepsilon}\right)$. For all $\mu>0$ small enough, choose $z_{\mu} \in C^{0}\left(Q_{\varrho_{0}, \delta}, \mathbb{R}^{+}\right)$such that:

- $\sup \left\{z_{\mu}(\varrho, \theta):(\varrho, \vartheta) \in Q_{\varrho_{0}, \delta}\right\} \leq \mu$;

- $z_{\mu}(\varrho, \vartheta)=\mu$ for $(\varrho, \vartheta) \in Q_{\varrho_{0}, \delta}$ such that $\left|\varrho-\varrho_{\varepsilon}\right|+\left|\vartheta-\vartheta_{\varepsilon}\right| \leq \mu / 2$;

- $z_{\mu}(\varrho, \vartheta)=0$ for $(\varrho, \vartheta) \in Q_{\varrho_{0}, \delta}$ such that $\left|\varrho-\varrho_{\varepsilon}\right|+\left|\vartheta-\vartheta_{\varepsilon}\right| \geq \mu$.

Since properties (2.8) hold and $u_{r, s, \varepsilon}^{ \pm} \neq 0$, it is easy to verify that there exists $\bar{\mu}>0$ small enough such that:

(2.14) $z_{\bar{\mu}}=0$ on the boundary of $Q_{\varrho_{0}, \delta}$;

(2.15) if $(\varrho, \vartheta) \in Q_{\varrho_{0}, \delta}$ satisfies $z_{\bar{\mu}}(\varrho, \vartheta)>0$ and if $\left.t \in\right] 0, z_{\bar{\mu}}(\varrho, \vartheta)[$, then $\sigma(\varrho, \vartheta)+t \bar{w} \in I\left(u_{r, s, \varepsilon}\right)$;

$$
\begin{aligned}
& \text { if } \left.\left.(\varrho, \vartheta) \in Q_{\varrho_{0}, \delta} \text { and } t \in\right] 0, z_{\bar{\mu}}(\varrho, \vartheta)\right], \\
& \text { then } \sigma(\varrho, \vartheta)+t \bar{w} \in K^{r, s} \text { and }[\sigma(\varrho, \vartheta)+t \bar{w}]^{ \pm} \not \equiv 0 .
\end{aligned}
$$

Since $f^{\prime}(u)[\bar{w}]<0$ for $u \in I\left(u_{r, s, \varepsilon}\right)$, if $(\varrho, \vartheta) \in Q_{\varrho_{0}, \delta}$ satisfies $z_{\bar{\mu}}(\varrho, \vartheta)>0$ and if $t \in] 0, z_{\bar{\mu}}(\varrho, \vartheta)[$, we have

Then

$$
\frac{\partial}{\partial t} f(\sigma(\varrho, \vartheta)+t \bar{w})<0
$$

$$
f\left(\sigma(\varrho, \vartheta)+z_{\bar{\mu}} \bar{w}\right) \leq f(\sigma(\varrho, \vartheta)) \quad \forall(\varrho, \vartheta) \in Q_{\varrho 0}, \delta
$$

and

$$
f\left(\sigma\left(\varrho_{\varepsilon}, \vartheta_{\varepsilon}\right)+z_{\bar{\mu}} \bar{w}\right)<f\left(\sigma\left(\varrho_{\varepsilon}, \vartheta_{\varepsilon}\right)\right) .
$$

As $\left(\varrho_{\varepsilon}, \vartheta_{\varepsilon}\right)$ is the unique maximum point of the function $f \circ \sigma$, it follows that

$$
\max \left\{f\left(\sigma(\varrho, \vartheta)+z_{\bar{\mu}}(\varrho, \theta) \bar{w}\right):(\varrho, \vartheta) \in Q_{\varrho_{0}, \delta}\right\}<f\left(u_{r, s, \varepsilon}\right) .
$$

Therefore, taking into account that

$$
\sigma(\varrho, \vartheta)+z_{\bar{\mu}}(\varrho, \vartheta) \bar{w} \in K^{r, s} \quad \forall(\varrho, \vartheta) \in Q_{\varrho_{0}, \delta}
$$


and that

$$
f\left(u_{r, s, \varepsilon}\right)=\min \left\{f(u): u \in H_{S}\left(\Omega_{\varepsilon}^{h}\right) \cap M \cap K^{r, s}\right\},
$$

we infer that

$$
\left\{\sigma(\varrho, \vartheta)+z_{\bar{\mu}}(\varrho, \vartheta) \bar{w}:(\varrho, \vartheta) \in Q_{\varrho_{0}, \delta}\right\} \cap M=\emptyset .
$$

On the other hand, if we set

$$
\varphi(u)=\frac{\|u\|_{p}^{p}}{\|D u\|_{2}^{2}} \quad \forall u \in H_{S}\left(\Omega_{\varepsilon}^{h}\right) \cap L^{p}\left(\Omega_{\varepsilon}^{h}\right) \text { with } u \not \equiv 0 \text { in } \Omega_{\varepsilon}^{h},
$$

the continuous function $\gamma: Q_{\varrho}, \delta \rightarrow \mathbb{R}^{2}$, defined by $\gamma(\varrho, \vartheta)=\left(\gamma_{1}(\varrho, \vartheta), \gamma_{2}(\varrho, \vartheta)\right)$ with

$$
\begin{aligned}
& \gamma_{1}(\varrho, \vartheta)=\varphi\left(\left[\sigma(\varrho, \vartheta)+z_{\bar{\mu}}(\varrho, \vartheta) \bar{w}\right]^{+}\right)+\varphi\left(\left[\sigma(\varrho, \vartheta)+z_{\bar{\mu}}(\varrho, \vartheta) \bar{w}\right]^{-}\right)-2, \\
& \gamma_{2}(\varrho, \vartheta)=\varphi\left(\left[\sigma(\varrho, \vartheta)+z_{\bar{\mu}}(\varrho, \vartheta) \bar{w}\right]^{+}\right)-\varphi\left(\left[\sigma(\varrho, \vartheta)+z_{\bar{\mu}}(\varrho, \vartheta) \bar{w}\right]^{-}\right),
\end{aligned}
$$

has the following properties:

(a) $\gamma_{1}(1, \vartheta) \geq 0$ for $\vartheta \in[\delta, \pi / 2-\delta]$,

(b) $\gamma_{1}\left(\varrho_{0}, \vartheta\right) \leq 0$ for $\vartheta \in[\delta, \pi / 2-\delta]$,

(c) $\gamma_{2}(\varrho, \delta) \geq 0$ for $\varrho \in\left[\varrho_{0}, 1\right]$,

(d) $\gamma_{2}(\varrho, \pi / 2-\delta) \leq 0$ for $\varrho \in\left[\varrho_{0}, 1\right]$.

In fact, as $z_{\bar{\mu}}=0$ on the boundary of $Q_{\varrho_{0}, \delta}$, (a) follows from (2.9), (b) from (2.11), (c) and (d) from (2.12) and (2.13).

Thus, applying a result of C. Miranda (see [26]), we deduce that there exists $(\bar{\varrho}, \bar{\vartheta}) \in Q_{\varrho_{0}, \delta}$ such that $\gamma(\bar{\varrho}, \bar{\vartheta})=0$, that is,

$$
\varphi\left(\left[\sigma(\bar{\varrho}, \bar{\vartheta})+z_{\bar{\mu}}(\bar{\varrho}, \bar{\vartheta}) \bar{w}\right]^{+}\right)=\varphi\left(\left[\sigma(\bar{\varrho}, \bar{\vartheta})+z_{\bar{\mu}}(\bar{\varrho}, \bar{\vartheta}) \bar{w}\right]^{-}\right)=1,
$$

which implies $\sigma(\bar{\varrho}, \bar{\vartheta})+z_{\bar{\mu}}(\bar{\varrho}, \bar{\vartheta}) \bar{w} \in M$, contrary to $(2.17)$.

\section{Qualitative properties of the solutions and concluding remarks}

In this section we describe the behaviour as $\varepsilon \rightarrow 0$ of the solutions $u_{r, s, \varepsilon}$ given by Theorem 2.2 (see Proposition 3.2). This behaviour implies, in particular, that different solutions correspond to different pairs $(r, s)$ for $\varepsilon>0$ small enough, so that we have $h^{2}$ solutions of $P\left(\Omega_{\varepsilon}^{h}, p\right)$. Moreover, we show that (unlike [21]) these solutions $u_{r, s, \varepsilon}$, which minimize $f$ on $H_{S}\left(\Omega_{\varepsilon}^{h}\right) \cap M \cap K^{r, s}$, are not local minima for $f$ on $M$ : indeed, we show that we cannot have local minima for $f$ on $M$ (see Remark 3.4).

Finally, we discuss some possible generalizations of the results obtained in this paper.

Notations 3.1. For all $\mu>0$, let $Q_{\mu}^{1}, \ldots, Q_{\mu}^{h}$ be the sets defined by

$$
Q_{\mu}^{i}=\left\{x=\left(x_{1}, \ldots, x_{n}\right) \in \mathbb{R}^{n}: \sum_{i=1}^{n-1} x_{i}^{2} \leq \mu^{2}, i \leq x_{n} \leq i+1\right\} \quad \forall i=1, \ldots, h .
$$


Proposition 3.2. For all $r, s=1, \ldots, h$ and $\varepsilon \in] 0, \bar{\varepsilon}[$ (see Lemma 2.3), let $u_{r, s, \varepsilon}$ be a solution of problem $P\left(\Omega_{\varepsilon}^{h}, p\right)$, minimizing $f$ in $H_{S}\left(\Omega_{\varepsilon}^{h}\right) \cap M \cap K^{r, s}$ (see Theorem 2.2). Then, for all $r, s=1, \ldots, h$, we have:

(a) $\lim _{\varepsilon \rightarrow 0^{+}} \sup \left\{u_{r, s, \varepsilon}^{+}(x): x \in \Omega_{\varepsilon}^{h} \backslash Q_{\mu}^{r}\right\}=0 \quad \forall \mu>0$, $\lim _{\varepsilon \rightarrow 0^{+}} \sup \left\{u_{r, s, \varepsilon}^{-}(x): x \in \Omega_{\varepsilon}^{h} \backslash Q_{\mu}^{s}\right\}=0 \quad \forall \mu>0$.

(b) $\lim _{\varepsilon \rightarrow 0^{+}} \sup \left\{u_{r, s, \varepsilon}^{+}(x): x \in \Omega_{\varepsilon}^{h} \cap Q_{\mu}^{r}\right\}=\infty \quad \forall \mu>0$, $\lim _{\varepsilon \rightarrow 0^{+}} \sup \left\{u_{r, s, \varepsilon}^{-}(x): x \in \Omega_{\varepsilon}^{h} \cap Q_{\mu}^{s}\right\}=\infty \quad \forall \mu>0$.

(c) $\lim _{\varepsilon \rightarrow 0^{+}}\left\|u_{r, s, \varepsilon}\right\|_{q}=0 \quad \forall q \leq p$.

(d) $\lim _{\varepsilon \rightarrow 0^{+}} \int_{\Omega_{\varepsilon}^{h} \cap Q_{\mu}^{r}}\left(u_{r, s, \varepsilon}^{+}\right)^{q} d x=\infty \quad \forall q>\frac{n}{2}(p-1), \quad \forall \mu>0$, $\lim _{\varepsilon \rightarrow 0^{+}} \int_{\Omega_{\varepsilon}^{h} \cap Q_{\mu}^{s}}\left(u_{r, s, \varepsilon}^{-}\right)^{q} d x=\infty \quad \forall q>\frac{n}{2}(p-1), \quad \forall \mu>0$.

For the proof it suffices to argue as in the proof of Propositions 2.11 and 3.11 of [35]. In particular, the proof of property (d) is based on the following proposition.

Proposition 3.3. Let $u$ be a solution of problem $P(\Omega, p)$ with $p>2$. Denote by $\lambda_{1}(\Omega)$ the first eigenvalue of the operator $-\Delta$ in $H_{0}^{1,2}(\Omega)$, that is,

$$
\lambda_{1}(\Omega)=\min \left\{\int_{\Omega}|D u|^{2} d x: u \in H_{0}^{1,2}(\Omega), \int_{\Omega} u^{2} d x=1\right\} .
$$

Then

$$
\sup _{\Omega} u^{ \pm} \geq\left[\lambda_{1}(\Omega)\right]^{1 /(p-2)} .
$$

Proof. Set

$$
\lambda_{1}^{+}=\min \left\{\int_{\Omega}|D v|^{2} d x: v \in H_{0}^{1,2}(\Omega), \int_{\Omega} v^{2} d x=1, \int_{\Omega}|v| u^{-} d x=0\right\} .
$$

Let $v^{+} \geq 0$ be a minimizing function for $\lambda_{1}^{+}$. Then

$$
\int_{\Omega} D v^{+} D w d x-\lambda_{1}^{+} \int_{\Omega} v^{+} w d x=0 \quad \forall w \in H_{0}^{1,2}(\Omega) \text { such that } \int_{\Omega}|w| u^{-} d x=0
$$

in particular, for $w=u^{+}$, we obtain

$$
\int_{\Omega} D v^{+} D u^{+} d x-\lambda_{1}^{+} \int_{\Omega} v^{+} u^{+} d x=0 .
$$

Notice that, obviously, $\lambda_{1}^{+} \geq \lambda_{1}(\Omega)$ and

$$
\int_{\Omega} D u^{+} D v^{+} d x-\int_{\Omega}\left(u^{+}\right)^{p-1} v^{+} d x=0
$$


because $u$ is a solution of $P(\Omega, p)$. Then we have

$$
\int_{\Omega}\left(\left(u^{+}\right)^{p-1}-\lambda_{1}^{+} u^{+}\right) v^{+} d x=0,
$$

while, if $\sup _{\Omega} u^{+}<\left[\lambda_{1}(\Omega)\right]^{1 /(p-2)}$, we should have

$$
\int_{\Omega}\left(\left(u^{+}\right)^{p-1}-\lambda_{1}^{+} u^{+}\right) v^{+} d x<0,
$$

because $\left(u^{+}(x)\right)^{p-1}<\lambda_{1}(\Omega) u^{+}(x) \leq \lambda_{1}^{+} u^{+}(x)$ for all $x \in \Omega$ satisfying $0<$ $u^{+}(x)<\left[\lambda_{1}(\Omega)\right]^{1 /(p-2)}$, and $v^{+}(x)>0$ if $u^{+}(x)>0$.

Analogous arguments hold for $u^{-}$.

REMARK 3.4. We already remarked that the minimum of $f$ on $H_{S}\left(\Omega_{\varepsilon}^{h}\right) \cap M$ is not achieved. We can also prove that $f$ does not even have local minimum points on $H_{S}\left(\Omega_{\varepsilon}^{h}\right) \cap M$; in particular, the solutions $u_{r, s, \varepsilon}$ given by Theorem 2.2, which minimize $f$ on $H_{S}\left(\Omega_{\varepsilon}^{h}\right) \cap M \cap K^{r, s}$, are not local minimum points for $f$ on $H_{S}\left(\Omega_{\varepsilon}^{h}\right) \cap M$.

In fact, for all $u \in H_{S}\left(\Omega_{\varepsilon}^{h}\right) \cap M$ we can find a sequence $\left(u_{i}\right)_{i} \in H_{S}\left(\Omega_{\varepsilon}^{h}\right) \cap M$ such that $u_{i} \rightarrow u$ in $L^{p}\left(\Omega_{\varepsilon}^{h}\right)$ and in $H_{0}^{1,2}\left(\Omega_{\varepsilon}^{h}\right)$ and, moreover, $f\left(u_{i}\right)<f(u)$ for all $i \in \mathbb{N}$. In order to get such a sequence, fix a nonnegative function $z \in$ $C_{0}^{\infty}(B(0,1))$ having radial symmetry with respect to the $x_{n}$-axis and such that $\int_{B(0,1)} z^{p} d x=1$. For all $\varrho>0$ set $z_{\varrho}(x)=z((x-\bar{x}) / \varrho)$ where $\bar{x}=\left(0, \ldots, 0, \bar{x}_{n}\right)$ with $0<\bar{x}_{n}<1-\sigma_{1}$ is a fixed point of the $x_{n}$-axis (see Notations 2.1).

For all $\varrho>0$ and $i \in \mathbb{N}$, let

$$
u_{i, \varrho}=\left(1-\frac{1}{i}\right)^{1 / p} u^{+}+\left(\frac{1}{i}\right)^{1 / p} \frac{\left\|u^{+}\right\|_{p}}{\left\|z_{\varrho}\right\|_{p}} z_{\varrho}-u^{-} .
$$

As in Remarks 2.13 and 3.12 of [35] one can verify that, for all $i \in \mathbb{N}$,

$$
\lim _{\varrho \rightarrow 0^{+}}\left\|u_{i, \varrho}^{ \pm}\right\|_{p}=\left\|u^{ \pm}\right\|_{p}
$$

and

$$
\begin{aligned}
\lim _{\varrho \rightarrow 0^{+}}\left\|D u_{i, \varrho}^{+}\right\|_{2}^{2} & =\left(1-\frac{1}{i}\right)^{2 / p}\left\|D u^{+}\right\|_{2}^{2}<\left\|D u^{+}\right\|_{2}^{2}, \\
\lim _{\varrho \rightarrow 0^{+}}\left\|D u_{i, \varrho}^{-}\right\|_{2}^{2} & =\left\|D u^{-}\right\|_{2}^{2} .
\end{aligned}
$$

Consequently, there exists an infinitesimal sequence $\left(\varrho_{i}\right)_{i}$ of positive numbers such that, if we set

$$
u_{i}=\left(\frac{\left\|D u_{i, \varrho_{i}}^{+}\right\|_{2}^{2}}{\left\|u_{i, \varrho_{i}}^{+}\right\|_{p}^{p}}\right)^{1 /(p-2)} u_{i, \varrho_{i}}^{+}-\left(\frac{\left\|D u_{i, \varrho_{i}}^{-}\right\|_{2}^{2}}{\left\|u_{i, \varrho_{i}}^{-}\right\|_{p}^{p}}\right)^{1 /(p-2)} u_{i, \varrho_{i}}^{-},
$$

the sequence $\left(u_{i}\right)_{i}$ in $H_{S}\left(\Omega_{\varepsilon}^{h}\right) \cap M$ has the desired properties. 
Notice that the nonexistence of local minimum points for $f$ on $H_{S}\left(\Omega_{\varepsilon}^{h}\right) \cap M$ shows, in particular, that the arguments used in [27], [28], [21], [31], based on the use of "barycentre functions" like

$$
\beta(u)=\frac{1}{\|u\|_{p}^{p}} \int_{\Omega_{\varepsilon}^{h}} x|u(x)|^{p} d x,
$$

cannot apply for $p>2^{*}$.

REMARK 3.5. The methods used in this paper can be applied to study problems with more general nonlinear terms. For example, analogous existence and multiplicity results can be proved for the nodal solutions of problems of the form

$$
P(\Omega, p, q)\left\{\begin{array}{l}
\Delta u+a\left(u^{+}\right)^{p-1}-b\left(u^{-}\right)^{q-1}=0, \\
u \in H_{0}^{1,2}(\Omega),
\end{array}\right.
$$

with $a, b \in \mathbb{R}^{+}$and $p, q \geq 2^{*}$.

REMARK 3.6. The domains $\Omega_{\varepsilon}^{h}$ considered in this paper do not have a smooth boundary; however, it is clear that the same methods can be used to state analogous results for smooth symmetric domains having a more general shape.

\section{REFERENCES}

[1] Adimurthi and S. L. Yadava, Elementary proof of the nonexistence of nodal solutions for the semilinear elliptic equations with critical Sobolev exponent, Nonlinear Anal. 14 (1990), 785-787.

[2] A. Ambrosetti And M. Struwe, A note on the problem $-\Delta u=\lambda u+u|u|^{2^{*}-2}$, Manuscripta Math. 54 (1986), 373-379.

[3] F. V. Atkinson, H. Brezis et L. A. Peletier, Solutions d'équations elliptiques avec exposant de Sobolev critique qui changent de signe, C. R. Acad. Sci. Paris Sér. I 306 (1988), 711-714.

[4] A. BAHRI AND J. M. CoRon, On a nonlinear elliptic equation involving the critical Sobolev exponent. The effect of the topology of the domain, Comm. Pure Appl. Math. 41 (1988), 253-294.

[5] H. BrezIs, Opérateurs maximaux monotones et semigroupes de contractions dans les espaces de Hilbert, North-Holland Math. Stud., vol. 5, North-Holland, Amsterdam, 1973.

[6] Elliptic equations with limiting Sobolev exponents. The impact of topology, Comm. Pure Appl. Math. 39 (1986), 17-39.

[7] H. Brezis And E. Lieb, A relation between pointwise convergence of functions and convergence of functionals, Proc. Amer. Math. Soc. 88 (1983), 486-490.

[8] H. Brezis and L. Nirenberg, Positive solutions of nonlinear elliptic equations involving critical Sobolev exponents, Comm. Pure Appl. Math. 36 (1983), 437-477.

[9] - A minimization problem with critical exponent and non zero data, Symmetry in Nature, Scuola Norm. Sup. Pisa, 1989, pp. 129-140.

[10] A. Capozzi, D. Fortunato and G. Palmieri, An existence result for nonlinear elliptic problems involving critical Sobolev exponent, Ann. Inst. H. Poincaré Anal. Non Linéaire 2 (1985), 463-470. 
[11] G. Cerami, D. Fortunato and M. Struwe, Bifurcation and multiplicity results for nonlinear elliptic problems involving critical Sobolev exponents, Ann. Inst. H. Poincaré Anal. Non Linéaire 1 (1984), 341-350.

[12] G. Cerami, S. Solimini And M. Struwe, Some existence results for superlinear elliptic boundary value problems involving critical exponents, J. Funct. Anal. 69 (1986), 289-306.

[13] G. Chobanov, A. Marino and D. Scolozzi, Evolution equation for the eigenvalue problem for the Laplace operator with respect to an obstacle, Rend. Accad. Naz. Sci. XL Mem. Mat. 14 (1990), 139-162.

[14] Multiplicity of eigenvalues for the Laplace operator with respect to an obstacle, and nontangency conditions, Nonlinear Anal. 15 (1990), 199-215.

[15] J. M. Coron, Topologie et cas limite des injections de Sobolev, C. R. Acad. Sci. Paris Sér. I 299 (1984), 209-212.

[16] E. N. Dancer, A note on an equation with critical exponent, Bull. London Math. Soc. 20 (1988), 600-602.

[17] W. Y. Ding, Positive solutions of $\Delta u+u^{(n+2) /(n-2)}=0$ on contractible domains, J. Partial Differential Equations 2 (1989), no. 4, 83-88.

[18] D. Fortunato and E. Jannelli, Infinitely many solutions for some nonlinear elliptic problems in symmetrical domains, Proc. Roy. Soc. Edinburgh Sect. A 105 (1987), 205213.

[19] P. L. Lions, The concentration-compactness principle in the calculus of variations. The limit case, Rev. Mat. Iberoamericana 1 (1985), no. 1, 145-201; no. 2, 45-121.

[20] M. V. MARChI, Nodal solutions of some elliptic problems with critical nonlinearities, Rend. Mat. Appl. 12 (1992), 99-125.

[21] M. V. Marchi AND F. Pacella, On the existence of nodal solutions of the equation $-\Delta u=|u|^{2^{*}-2} u$ with Dirichlet boundary conditions, preprint, Dip. Mat. Univ. Roma "La Sapienza".

[22] F. Merle and L. A. Peletier, Asymptotic behaviour of positive solutions of elliptic equations with critical and supercritical growth. I. The radial case, Arch. Rational Mech. Anal. 112 (1990), 1-19.

[23] Asymptotic behaviour of positive solutions of elliptic equations with critical and supercritical growth. II. The nonradial case, J. Funct. Anal. 105 (1992), 1-41.

[24] Positive solutions of elliptic equations involving supercritical growth, Proc. Roy. Soc. Edinburgh 118 (1991), 49-62.

[25] - On supercritical phenomena, Nonlinear Diffusion Equations and their Equilibrium States, 3 (Gregynog, 1989), Progr. Nonlinear Differential Equations Appl., vol. 7, Birkhäuser, 1992, pp. 417-424.

[26] C. Miranda, Un'osservazione su un teorema di Brouwer, Boll. Un. Mat. Ital. (2) 3 (1940), 5-7.

[27] D. Passaseo, Multiplicity of positive solutions of nonlinear elliptic equations with critical Sobolev exponent in some contractible domains, Manuscripta Math. 65 (1989), 147166.

[28] The effect of the domain shape on the existence of positive solutions of the equation $-\Delta u+u^{2^{*}-1}=0$, Topol. Methods Nonlinear Anal. 3 (1994), 27-54.

[29] Molteplicità di soluzioni per certe disequazioni variazionali di tipo ellittico, Boll. Un. Mat. Ital. B (7) 3 (1989), 639-667.

[30] _ Molteplicità di soluzioni per disequazioni variazionali nonlineari di tipo ellittico, Rend. Accad. Naz. Sci. XL Mem. Mat. 15 (1991), 19-56.

[31] Su alcune successioni di soluzioni positive di problemi ellittici con esponente critico, Atti Accad. Naz. Lincei Rend. (9) Mat. Appl. 3 (1992), 15-21. 
[32] Some sufficient conditions for the existence of positive solutions to the equations $-\Delta u+a(x) u=u^{2^{*}-1}$ in bounded domains, Ann. Inst. H. Poincaré Anal. Non Linéaire 13 (1996), 185-227.

[33] Nonexistence results for elliptic problems with supercritical nonlinearity in nontrivial domains, J. Funct. Anal. 14 (1993), 97-105.

[34] - New nonexistence results for elliptic equations with supercritical nonlinearity, Differential Integral Equations 8 (1995), 577-586.

[35] _ Esistenza e molteplicità di soluzioni positive per equazioni ellittiche con nonlinearità sopracritica, Rend. Accad. Naz. Sci. XL Mem. Mat. 16 (1992), 77-98.

[36] S. I. Poкhozhaev, Eigenfunctions of the equation $\Delta u+\lambda f(u)=0$, Soviet Math. Dokl. 6 (1965), 1408-1411.

[37] P. Rabinowitz, Some global results for nonlinear eigenvalue problems, J. Funct. Anal. 7 (1971), 487-513.

[38] O. ReY, Sur un problème variationnel non compact: l'effet de petits trous dans le domaine, C. R. Acad. Sci. Paris Sér. I 308 (1989), 349-352.

[39] S. Solimini, On the existence of infinitely many radial solutions for some elliptic problems, Rev. Mat. Apl. 9 (1987), 75-86.

[40] M. Struwe, A global compactness result for elliptic boundary value problems involving limiting nonlinearities, Math. Z. 187 (1984), 511-517.

[41] G. Talenti, Best constant in Sobolev inequality, Ann. Mat. Pura Appl. 110 (1976), $353-372$.

[42] G. Tarantello, Nodal solutions of semilinear elliptic equations with critical exponent, Differential Integral Equations 5 (1992), 25-42.

[43] D. ZhANG, On multiple solutions of the equation $\Delta u+\lambda u+|u|^{4 /(n-2)} u=0$, Nonlinear Anal. 13 (1989), 353-372.

Donato Passaseo

Dipartimento di Matematica

Università di Pisa

Via Buonarroti, 2

56127 Pisa, ITALY 